\title{
Concepções dos pais acerca da doença oncológica e do tratamento quimioterápico de seus filhos
}

Parents' conceptions on their children's oncologic condition and the chemotherapy

Concepciones de los padres acerca de la enfermedad oncológica y del tratamiento quimioterápico de sus

hijos

\section{Fernanda Machado Silva-Rodrigues ${ }^{\mathrm{I}}$, Verônica Leticia da Silva Vulczak ${ }^{\mathrm{II}}$, Carolina Fonseca Alencar $^{\mathrm{III}}$, Luciana Soares Costa Santos ${ }^{\mathrm{IV}}$, Lucila Castanheira Nascimento ${ }^{\mathrm{V}}$}

\begin{abstract}
Resumo: Objetivo: analisar as concepções de pais e mães de crianças com câncer em relação à doença e ao tratamento quimioterápico de seus filhos, de acordo com os conceitos do Modelo do Senso Comum (MSC). Método: estudo descritivo, qualitativo. Foram entrevistados 14 pais de pacientes pediátricos em tratamento oncológico. Os dados foram submetidos à análise de conteúdo e analisados segundo o MSC. Resultados: os resultados foram reunidos nas categorias: Identidade da doença: concepções sobre o câncer; Duração: o tempo para o diagnóstico e os sintomas envolvidos; Causas da doença: a busca por explicações; Consequências da doença e do tratamento; Cura ou controle: percepções sobre o prognóstico, baseadas nos domínios do MSC. Conclusão: concepções negativas relacionadas ao câncer e seu tratamento podem gerar incertezas nos pais, comprometer a mobilização de recursos para lidar com os desdobramentos da doença e reduzir as expectativas de um desfecho positivo da experiência.
\end{abstract}

Descritores: Neoplasias; Criança; Adolescente; Pais

Abstract: Objective: analyze the conceptions of fathers and mothers of children with cancer about their children's disease and chemotherapy, in accordance with the concepts of the Commonsense Model (CSM). Method: descriptive, qualitative study. Fourteen parents of pediatric patients in cancer treatment were interviewed. The data were submitted to content analysis and analyzed according to the CSM. Results: the results were joined in the categories: Identity of the disease: conceptions on the cancer; Duration: time for the diagnosis and symptoms involved; Causes of the disease: the search for explanations; Consequences of the disease and the treatment; Cure or control: perceptions on the prognosis, based on the domains of the CSM. Conclusion: negative conceptions on

\footnotetext{
I Enfermeira. Doutora. Docente do Curso de Graduação em Enfermagem da Faculdade de Ciências Médicas da Santa Casa de São Paulo (FCMSCSP), São Paulo - SP, Brasil. E-mail: fernanda.rodrigues@fcmsantacasasp.edu.br, ORCID: https://orcid.org/0000-0002-8412-2333

II Enfermeira. Egressa do Curso de Graduação em Enfermagem da Faculdade de Ciências Médicas da Santa Casa de São Paulo (FCMSCSP), São Paulo - SP, Brasil. E-mail: leticia_vulczak@hotmail.com, ORCID: https://orcid.org/0000-0003-0268-0436

III Enfermeira. Egressa do Curso de Graduação em Enfermagem da Faculdade de Ciências Médicas da Santa Casa de São Paulo (FCMSCSP), São Paulo - SP, Brasil. E-mail: fonseca.alencar@yahoo.com.br, ORCID: https://orcid.org/0000-0002-1332-347X

IV Enfermeira. Doutora. Docente do Curso de Graduação em Enfermagem da Faculdade de Ciências Médicas da Santa Casa de São Paulo (FCMSCSP), São Paulo - SP, Brasil. E-mail: luciana.santos@fcmsantacasasp.edu.br, ORCID: https://orcid.org/0000-0001-5708-1460

v Enfermeira. Doutora. Professora Titular do Departamento de Enfermagem Materno-Infantil da Escola de Enfermagem de Ribeirão Preto - EERP, da Universidade de São Paulo (USP), Ribeirão Preto - SP, Brasil. E-mail: lucila@eerp.usp.br, ORCID: https://orcid.org/0000-0002-7900-7111
} 
the cancer and its treatment can arouse uncertainties in the parents, compromise the mobilization of resources to cope with the consequences of the disease and reduce the expections that the experience will have a positive outcome.

Descriptors: Neoplasms; Child; Adolescent; Parents

Resumen: Objetivo: analizar las concepciones de los padres de niños con cáncer respecto a la enfermedad y al tratamiento quimioterápico de sus hijos, según los conceptos del Modelo del Sentido Común (MSC). Método: estudio descriptivo, cualitativo. Fueron entrevistados 14 padres de niños en tratamiento oncológico. Los datos fueron sometidos al análisis de contenido y analizados según el MSC. Resultados: los resultados fueron reunidos en las categorías: Identidad de la enfermedad: concepciones sobre el cáncer; Duración: el tiempo para el diagnóstico y los síntomas involucrados; Causas de la enfermedad: la búsqueda por explicaciones; Consecuencias de la enfermedad y del tratamiento; Cura o control; percepciones sobre el pronóstico, basadas en los dominios del MSC. Conclusión: concepciones negativas relacionadas al cáncer y su tratamiento pueden generar incertidumbres, comprometer la movilización de recursos para lidiar con los desdoblamientos de la enfermedad y reducir las expectativas de un desfecho positivo de la experiencia.

Descriptores: Neoplasias; Nino; Adolescente; Padres

\section{Introdução}

O câncer infantojuvenil representa a primeira causa de morte por doenças na faixa etária de zero a 19 anos, com uma média de 12.600 casos diagnosticados por ano no Brasil. ${ }^{1}$ As neoplasias mais comuns na infância são as leucemias, principalmente, as leucemias agudas, os tumores do Sistema Nervoso Central (SNC) e os linfomas. ${ }^{2}$

O câncer sempre foi associado à ideia de morte, dor, incerteza, confusão e impotência, tanto para os pacientes quanto para as famílias. ${ }^{3}$ Quando a doença acomete uma criança, consequentemente, o impacto abrange toda a estrutura familiar e social. Observam-se inversões nos papéis familiares, mudanças na rotina, reformulação dos objetivos de vida, das expectativas e da esperança para os envolvidos. Alguns pais recorrem a explicações não científicas a fim de compreenderem as possíveis causas do adoecimento de seus filhos. ${ }^{3}$

As concepções do indivíduo são desenvolvidas de forma variada e podem ser influenciadas pelo nível de instrução, meio onde vivem e cultura, sendo, portanto, mutáveis. ${ }^{4}$ Podem determinar a capacidade das pessoas para apreender uma ideia ou questão e de perceber um fenômeno, estabelecendo seu próprio ponto de vista. ${ }^{5}$ 
3 | Silva-Rodrigues, FM; Vulczak VLS; Alencar CF; Santos LSC; Nascimento LC

A literatura destaca que pacientes e familiares podem acreditar que a ocorrência de efeitos adversos do tratamento é uma evidência de sua eficácia. Assim, maiores danos e sofrimento implicariam em melhores respostas e maior esperança de sobrevivência. Já a ausência desses efeitos secundários à terapia gera incertezas, ansiedade e questionamentos em relação ao êxito do tratamento. ${ }^{6}$

Alguns pais de crianças em quimioterapia compreendem que as drogas atingem tanto as células boas quanto ruins e têm ciência de que no início os sintomas são piores, mas que com o passar do tratamento, o paciente pode dispor de recursos para minimizar esses efeitos desagradáveis. Para outros, no entanto o tratamento é visto como prejudicial e, ao invés de ajudar, causa limitações piores que a própria doença ${ }^{6-7}$

O adoecimento de um filho por câncer representa uma ameaça e pode causar representações sobre a doença, influenciadas por crenças individuais. Essas crenças podem comprometer a aceitação da doença e tratamento, os comportamentos e o enfrentamento da situação vivida. ${ }^{8}$ Esse modo de representação da doença é denominado de Modelo do Senso Comum (MSC) ou Modelo de Autorregulação do Comportamento em Saúde, ${ }^{8}$ referencial adotado para este estudo.

Segundo o MSC a percepção da doença pode ser compreendida considerando cinco dimensões: identidade - expressa por rótulos abstratos, como o nome da doença e sintomas associados a ela; causas - fatores relacionados à origem da doença; duração - duração temporal ou curso da doença ao longo do tempo (por exemplo, aguda, crônica ou cíclica); consequências gravidade da doença e seu impacto no funcionamento físico, psicológico e social e controle/cura - possibilidade de curar ou controlar a doença. ${ }^{8}$

Partindo-se do exposto, pergunta-se: quais as concepções de pais e mães de crianças e adolescentes com câncer sobre a doença e tratamento de seus filhos? A partir dessa questão, 
estabeleceu-se como objetivo, analisar as concepções de pais e mães de crianças com câncer em relação à doença e ao tratamento quimioterápico de seus filhos, de acordo com os conceitos do MSC.

\section{Método}

Trata-se de um estudo descritivo, qualitativo, com discurso examinado à luz do MSC ou Modelo de Autorregulação do Comportamento em Saúde. A coleta dos dados foi realizada no ambulatório de quimioterapia de um hospital referência no tratamento do câncer infantojuvenil em São Paulo - SP, entre os meses de junho a agosto de 2016. Excluíram-se os pais de crianças ou adolescentes cujos filhos recebiam quimioterapia paliativa ou encontravam-se fora de possibilidades terapêuticas curativas. Os participantes integraram o estudo por conveniência e não houve recusa quanto à participação.

Foram realizadas entrevistas semiestruturadas, áudio-gravadas, orientadas por um roteiro previamente elaborado pelas autoras, com base na literatura sobre o tema. As questões referiamse à compressão conceitual dos pais a respeito da doença de seus filhos, além de abordarem a ação dos quimioterápicos no organismo da criança na perspectiva dos pais. Foi necessário apenas um encontro com cada participante. Os depoimentos obtidos nas entrevistas foram transcritos na íntegra para melhor compreensão dos discursos e submetidos aos procedimentos da análise de conteúdo dedutiva. A coleta dos dados foi interrompida quando o conjunto de dados obtidos nas entrevistas se mostrou redundante e suficiente para a compreensão do fenômeno em estudo, de acordo com a matriz estruturada, baseada no MSC. ${ }^{9}$

A modalidade dedutiva é empregada quando a estrutura de análise é operacionalizada com base em conhecimento e conceitos prévios. ${ }^{9}$ Por meio dela, os pesquisadores têm o intuito de testar categorias, conceitos, modelos ou hipóteses pré-existentes. Tanto a análise indutiva, quanto a dedutiva dividem-se em três fases principais: preparação, organização e relato dos resultados. $\mathrm{Na}$ fase de preparação, foi realizada a leitura exaustiva do material empírico, 
5 | Silva-Rodrigues, FM; Vulczak VLS; Alencar CF; Santos LSC; Nascimento LC

identificando palavras, frases e expressões que respondessem ao objetivo do estudo. Em seguida, os dados foram organizados segundo uma matriz estruturada, baseada nas cinco dimensões do modelo teórico adotado (MSC): identidade, causas, duração, consequências e controle/cura. Assim, apenas aspectos que se ajustaram às cinco dimensões do modelo foram selecionados dos depoimentos dos participantes, originando as categorias apresentadas nos resultados. O estudo atendeu as exigências da Resolução 466/12 do Conselho Nacional de Saúde e foi submetido ao Comitê de Ética em Pesquisa da instituição sob o número CAAE 56098216.4.0000.5479, sendo aprovado sob o parecer $\mathrm{n}^{\circ} 1.561 .355$, em 25 de maio de 2016.

\section{Resultados e discussão}

A amostra foi composta por 14 pais, sendo 13 mães $(92,8 \%)$ e um pai $(7,2 \%)$, com idade média de 39,9 anos, a maioria com ensino médio (71,4\%) e desempregados (78,5\%). Quanto à profissão, àqueles inseridos no mercado de trabalho, declararam-se terapeuta, psicóloga e costureira, representado $7,1 \%$ cada. Em relação ao diagnóstico das crianças, os mais predominantes foram tumores ósseos e linfoma com 21,4\% de casos, cada, seguidos por leucemias, meduloblastoma e tumores de sistema nervoso central, com $14,2 \%$ cada. Outros tipos de tumores como sarcoma e germinona também foram encontrados, totalizando 14,6\%. A seguir, apresentam-se os resultados da análise dedutiva, sintetizados em categorias:

\section{Identidade da doença: concepções sobre o câncer}

Nas entrevistas, os participantes relataram sua percepção sobre o diagnóstico de seus filhos, a partir de seus conhecimentos prévios sobre a doença. Baseados em pré-concepções, os pais usaram os seguintes termos para caracterizar o câncer: susto, monstro, doença danada, terrível, maligna, desesperadora, sinônimo de morte, terrível, devastadora e agressiva. Portanto, nota-se que, a doença, sendo ela de bom ou mau prognóstico, está associada a denominações estigmatizadas. 
Concepções dos pais acerca da doença oncológica e do tratamento quimioterápico... 6

O câncer é um susto! A gente vem de uma cidade bem pequena, quando foi diagnosticado com tumor na cabeça a gente já pensa o pior. Eu ainda brinco, falo que é o fundo do poço e lá no fundo tem um ralo para descer mais um pouquinho. Mas, quando você descobre a tempo, tem cura. (E1)

Quando descobri a doença dele [criança], foi um dia bem difícil, fiquei bastante revoltada com Deus por ter deixado isso acontecer, e sinceramente, para mim, câncer é sinônimo de morte, quando soube pensei todas as coisas ruins que podiam acontecer, nada positivo. Sempre tive muito medo. Na minha terra, no Pernambuco, nem a palavra câncer se falava. (E5)

O câncer é uma doença estigmatizada, permeada por concepções negativas e frequentemente associada à letalidade. Um estudo brasileiro mostrou impressões da população leiga sobre o câncer e apontou os termos mais associados à doença como a morte, dor e sofrimento. ${ }^{10}$

Assim, como descrito em outro estudo, com pais e mães de crianças com câncer acerca de seu conhecimento e atitudes frente à doença de seus filhos, observou-se que as dificuldades relacionadas à busca por informação, pelos pais sobre a doença e sintomas de seus filhos estavam diretamente ligadas às concepções negativas e ao estigma da doença na sociedade. ${ }^{3}$ Muitos pais evitavam sequer mencionar a palavra câncer, pois isto remetia às experiências negativas associadas à doença, seja por conceitos prévios ou pela perda de familiares acometidos por doenças oncológicas.

Além do estigma da doença, existe ainda um padrão paternalista na relação médicopaciente em nossa sociedade, que impõe barreiras para a maioria dos pais falarem abertamente sobre a doença e o tratamento, uma vez que eles confiam plenamente nos conhecimentos científicos e decisões da equipe médica. ${ }^{10}$

As concepções de pais e mães sobre a doença e tratamento mostraram-se permeadas por mitos e estigmas, os quais devem ser esclarecidos pelos profissionais de saúde, sobretudo, os de 
enfermagem. O estigma pode ter um efeito silenciador e afetar o comportamento dos indivíduos e seu modo de vida, além de tornar difícil lidar com o diagnóstico e tratamento. ${ }^{10-11}$ É necessário que a equipe multidisciplinar esteja preparada para, no momento do diagnóstico, trabalhar as concepções, dúvidas e as questões que acharem importantes para o esclarecimento dos anseios e questionamentos dos familiares. É importante que seja explicado o significado da palavra câncer, e que a família não teve participação ou culpa no desenvolvimento da doença. ${ }^{11-12}$

\section{Duração: o tempo para o diagnóstico e os sintomas envolvidos}

Com a análise dos discursos foi possível perceber que tanto as crianças, quanto os pais perceberam que algo não estava bem e pelos sinais e sintomas apresentados, decidiram, então, procurar o serviço de saúde.

Demorou para descobrir o que ele [adolescente] tinha, foi uma luta, fazia exames e os médicos achavam que era "íngua”, começou com um caroço no pescoço, ele fez ultrassom, tomografia, e que essa "íngua” era de uma infecção que não tinha surgido ainda, os exames dava tudo normal, até que fez a biópsia e deu linfoma no pescoço, deu tumor maligno. (E9)

Foi diagnosticado agora em janeiro, nós ficamos dois meses indo em pronto socorro e ninguém descobria o que ele [criança] tinha, sempre era diagnosticado com uma infecção, mas nenhum médico observou realmente os sintomas que ele estava, saía sangue do nariz, plaquetas baixa, hematomas, febre contínua, dor nas articulações, tudo acompanhado com infecção da garganta e imunidade baixa. Os testículos dele aumentaram, tinha infecções recorrentes e palidez. (E6)

O diagnóstico precoce do câncer infantil é um desafio para a medicina. ${ }^{13} \mathrm{Na}$ maioria das vezes, a associação dos sintomas com a doença não é aparente. Assim, boa parte dos pacientes pediátricos chega às instituições especializadas com a doença em estágios avançados, devido à dificuldade dos médicos em reconhecer sintomas inespecíficos, além da falta de informação dos pais e medo do diagnóstico. Na maioria das vezes, os sinais e sintomas do câncer infantil por não se mostrarem específicos, podem ser confundidos com os de outras doenças comuns da 
infância. ${ }^{14}$ Os fatores relacionados às dificuldades na elucidação diagnóstica, somados ao longo itinerário até a descoberta da doença e início do tratamento podem influenciar no prognóstico, comprometendo a sobrevida e as possibilidades de cura da doença. ${ }^{15}$

Diante disso, percebe-se a inadequação na manutenção do fluxo de atendimento, já apontados na literatura, que destaca o suporte comprometido na avaliação da efetividade dos serviços, como garantia do acesso e atendimento das necessidades da população. Nesse contexto, o enfermeiro deve buscar um olhar atentivo e uma escuta qualificada, para as crianças com sinais e sintomas sugestivos de câncer, favorecendo, assim o diagnóstico precoce dessa doença. ${ }^{15}$

\section{Causas da doença: a busca por explicações}

Os depoentes mostraram em seus relatos que não encontraram ligações causais para a doença de seus filhos, levando em consideração o pré-natal, nascimento bem-sucedido e o modo de vida prévio ao adoecimento. Por outro lado, outros participantes, mostraram não querer pensar em como a patologia se desenvolveu, uma vez que a pressão imposta pela situação já causa um estresse e uma avalanche de emoções desgastante o suficiente.

Sempre foi saudável, nasceu perfeito, teve uma gestação tranquila, um prénatal tranquilo. (E13)

Não gosto nem de pensar sobre isso, eu procuro abstrair, eu evito entrar nesse assunto, pois eu sempre preciso estar forte para estar com ele, se eu entrar nessa questão do que é, e do porquê aconteceu, eu piro, então, prefiro viver um dia de cada vez, e não gosto de falar sobre isso. (E6)

As doenças oncológicas, apesar de atingirem adultos e crianças, parecem impactar mais a sociedade ao acometerem crianças e adolescentes, como identificado no depoimento.

É uma doença cruel, independentemente da idade, para um adulto já é difícil, imagina para uma criança, uma criança não sabe lidar com o que está acontecendo, e é um tratamento bem desgastante, bem sofrido. (E13) 
A criança apresenta mais anos de potencial de vida e por isso é tida como um ser frágil. A ideia de doença e morte na infância é algo que contradiz com a ordem natural dos acontecimentos e o luto devido à existência de uma doença maligna na criança ou adolescente pode ser experimentado mais intensamente, além de levar os pais a refletirem e questionarem o porquê da doença acontecer exatamente com seus filhos e em sua família.,16

Quando questionados sobre sua percepção em relação ao que causou o câncer de seus filhos, os participantes relataram:

teve uma doença que era "parente" da catapora que ele [criança] pegou não lembro o nome, e isso foi o começo de tudo, nós ficamos desesperados. (E6)

ele [Deus] é que deixou tudo isso acontecer, mas ainda haverá vida, para deixar-nos mais fortes. Vejo isso como um processo, não como uma penalidade, um castigo, eu vejo isso como uma prova que Deus deu uma missão para a gente passar, pois a gente consegue passar. (E13)

Os pais explicaram por meio de seus relatos, situações como a absorção da radiação durante a gravidez, doenças maltratadas, exposição a substâncias químicas ou vacinas, associados ao enfraquecimento do sistema imunológico, facilitando, assim, o aparecimento da doença. Alguns ainda estabeleceram uma relação entre o câncer e traumas esportivos do passado. Percebe-se que os pais tentaram estabelecer causalidade tanto sob a perspectiva biomédica quanto à sobrenatural. Estudo sobre temática semelhante apontou que os pais se basearam em uma visão fatalista para entender os motivos que causaram a doença de seus filhos, ou seja, baseada na vontade de Deus, seja para testar a fé desses indivíduos ou para mostrar sua soberania e controle frente a situações adversas. ${ }^{17}$ Os resultados corroboram com outros achados na literatura, ${ }^{4-6}$ em que o adoecimento não parece algo passível do controle humano e pode ser interpretado como uma missão a ser cumprida, como prova de fé.

Essa interpretação sobrenatural dos fatos pode aliviar a culpa dos pais pelo adoecimento da criança, já que, se o divino controla os acontecimentos, logo são menores as possibilidades de 
interveniência e culpa. Por outro lado, podem desencadear a culpa relacionada ao não cumprimento de regras de fé e representarem punição por má conduta espiritual. ${ }^{18}$ Sabe-se que a visão estritamente biomédica, se apoia na resolução da doença, exclusivamente pela remediação de suas causas, numa visão sobrenatural ou miraculosa, tendendo a levar os pais a conceberem conceitos errados sobre o adoecimento e acerca da terapêutica, o que poderá repercutir em seu enfrentamento e adaptação diante de todos os acontecimentos contrários às suas expectativas. ${ }^{12,18}$ A busca por uma explicação lógica para as causas da doença é inconsciente, já que as famílias buscam entender as razões por que passam por acontecimentos tão penosos. Para os pais, o adoecimento é caracterizado por intenso sofrimento de seus filhos, acompanhado por incertezas, as quais oscilam entre o medo da morte e a esperança de cura. ${ }^{15}$

\section{Consequências da doença e do tratamento}

Após aceitarem o diagnóstico e passarem da fase de esquivamento para a fase de enfrentamento da doença, os pais vislumbram a possibilidade de cura para a doença e tendem a aceitar melhor o tratamento.

Quando a gente vem para um lugar que tem recurso, você fica mais calma, entende o que está acontecendo, o tratamento que vai ser realizado, e as consequências, as coisas começam a ficar mais leves. (E3)

O principal tratamento para o câncer infantil é a quimioterapia, tratamento farmacológico que tem por objetivo interferir com a capacidade de crescimento e reprodução das células, pode ser usada sozinha ou em conjunto com outras terapias, tais como radiação ou cirurgia. ${ }^{4}$ Parte dos participantes mostrou compreender os efeitos da quimioterapia no organismo de seus filhos, como expresso nos trechos:

acho que ela [quimioterapia] age tanto nas células boas quanto nas ruins, a quimioterapia que ele está fazendo hoje, me disseram que é mais especifica, age mais pegando as células mais resistentes. (E4) 
ela destrói as células ruins que são as células cancerígenas e as células boas também. Cada organismo responde de uma maneira, o meu de uma maneira o seu de outra, o dela de outro. (E1)

eu sei que ela se sente mal, mas é um bem para ela, é o remédio que vai fazer o tumor diminuir para haver uma cura o quanto antes. (E2)

A quimioterapia pode ser bastante eficaz no tratamento de certos tipos de câncer, porém assim como é de conhecimento de alguns participantes, os agentes não diferenciam as células saudáveis normais de células cancerosas, o que pode levar a muitos efeitos adversos durante o tratamento, variáveis de acordo com o fármaco utilizado. Os sintomas e eventos adversos mais comuns em crianças e adolescentes são: náuseas, vômitos, fadiga, alopecia, sangramentos, imunossupressão, anorexia, entre outros. ${ }^{4}$

Nas entrevistas pode-se perceber que a fase do tratamento e as consequências que o mesmo traz não se refletem apenas na criança e sim na família toda, pois há uma mudança de rotina extremamente grande.

É um tratamento intensivo, desgastante, a gente chega aqui cedo e vai embora só a noite, mudou nossa rotina de vida. (E8)

Como ele era um adolescente que andava de bicicleta, jogava bola e empinava pipa, ele parou, então você percebe que a pessoa não está bem, ele ficava o dia inteiro em casa, jogando vídeo game, deitado na cama e mesmo assim com muita dor, quando ele melhorava um pouco ia na rua soltar pipa, mas quando era noite, parece que voltava bem pior. (E11)

Nota-se que os entrevistados mostraram compreensão divergente acerca da quimioterapia. Na perspectiva deles, da mesma forma que o tratamento faz o bem, também faz o mal, como descrito nos trechos:

eu sei que ela se sente mal, mas é um bem para ela, é o remédio que vai fazer o tumor diminuir para haver uma cura o quanto antes. (E2) 
Concepções dos pais acerca da doença oncológica e do tratamento quimioterápico... 12

toda doença você toma um remédio e a tendência é diminuindo e a pessoa se fortalecendo, o câncer é ao contrario o remédio tem uma reação muito ruim, acaba com as células ruins e as boas. (E3)

destrói tudo de bom e de ruim, é um veneno bom, pois acaba tudo que é bom, mas está tratando as coisas ruins, destrói a criança, mas precisa, essa é a cura, não tem outro jeito. (E7)

Os participantes mostraram compreender a ação das drogas nas células tumorais e ainda ressaltaram os sintomas e eventos adversos causados por tais medicações nas células saudáveis do organismo da criança:

Faz mal, nada de bem isso aqui faz, por exemplo, você toma hoje MTX [Metotrexate] se ele não por tudo para fora em alguns dias, isso prejudica o rim, fígado, todos os órgãos, isso faz bem, me explica? Mata célula ruim e mata a célula boa. (E12)

A quimioterapia diminuiu bastante [tumor], e nesse intervalo todo não sentia mais dor. Só teve uma intercorrência, ficou seis dias sem comer por causa da quimioterapia, tinha dificuldade de comer e beber água, ficou muito fraco e desidratado, passou mal. (E11)

A literatura aponta que muitos pacientes e familiares associam os sintomas a ação efetiva da quimioterapia, ou seja, quanto mais intensos os sintomas apresentados, maiores os possíveis efeitos da quimioterapia no organismo e, consequentemente, melhor a resposta ao tratamento. ${ }^{6}$ A quimioterapia caracteriza-se como um tratamento que traz dor e sofrimento, mas ao mesmo tempo, materializa a luta contra a doença e instrumentaliza os pais a acreditarem na melhora clínica e possível cura da criança. $\cdot^{6-7}$

\section{Cura ou controle: percepções sobre prognóstico}

O conceito de cura é frequentemente associado à espiritualidade, uma vez que um diagnóstico como o câncer, causa sentimento de perda e luto antecipado, por parte dos familiares. A incapacidade de lidar com as situações impostas pela doença diminui a esperança. Dessa forma, a espiritualidade vem sendo estudada e se mostra um importante recurso na 
13 | Silva-Rodrigues, FM; Vulczak VLS; Alencar CF; Santos LSC; Nascimento LC

perspectiva individual, em situações adversas e como apoio para geração de menores taxas de ansiedade, diminuição do estresse, aumento da esperança e satisfação com os resultados do tratamento. $^{18}$

Depois que meu filho começou a tomar, ele se transformou, de como ele chegou no hospital para agora nossa ele se transformou. É um milagre, eu agradeço a esse santo remédio que parece que faz tanto mal, mas faz bem. (E9)

Depois que eu vim para cá e minha filha começou o tratamento, eu vi que o câncer tem cura sim, hoje em dia a tecnologia está muito avançada, e, primeiramente, Deus depois os médicos. Aonde os médicos não conseguem colocar a mão, Deus chega e cura, para mim o câncer tem cura sim antes eu não acreditava. (E4)

Hoje eu acredito na cura. Sempre tive Fé em Deus, mas quando se passa por problemas a fé acaba aumentando. (E6)

A gente tem um tratamento eficaz, um tratamento que vem mostrando resultado, que as pesquisas vêm crescendo cada vez mais, mas ainda não tem uma solução definitiva. Nossa fé é muito grande. (E13)

A espiritualidade pode ser descrita como um suporte que aumenta a relação entre a esperança e o enfrentamento em relação à doença, graças ao suporte promovido pela fé, frente aos acontecimentos. Para alguns pais, crenças e práticas espirituais foram adotadas a partir de uma situação estressante (diagnóstico do câncer de seus filhos), o que modificou a experiência associada ao sofrimento, configurando-se, assim, em uma forma de encontrar consolo, conforto e esperança. ${ }^{19}$ Sendo assim, a espiritualidade manifesta-se como importante estratégia de enfrentamento durante a trajetória da doença, possibilitando a busca do significado e propósito de vida/cura dos pacientes. Muitos familiares consideram a espiritualidade como um importante recurso durante o tratamento contra o câncer, sem minimizar a importância da continuidade da terapia convencional. ${ }^{18-19}$

\section{Considerações finais}


As concepções de pais e mães sobre o adoecimento de seus filhos, interpretadas à luz do MSC, descreveram o câncer infantil como uma ameaça que gerou medo, ansiedade e outras emoções, frente as quais a negação aparece como estratégia de enfrentamento em curto prazo, proporcionando alívio temporário. Entretanto, a negação ou não aceitação da doença em longo prazo, dificulta a elaboração de emoções, repercutindo negativamente no preparo desses pais para lidar com os desafios impostos pela doença e seu tratamento. Para os participantes, a doença e o tratamento se mostraram ambivalentes, ou seja, simultaneamente permeadas por medo e pela esperança de melhora e cura. O tratamento quimioterápico destacou-se como um mal necessário, cujos benefícios tenderam a sobrepor os eventos adversos.

Apesar das limitações do estudo, concernentes à realização da coleta de dados em um único centro, seus resultados descrevem as concepções dos pais relacionadas à doença de seus filhos. Munidos dessas informações, esses profissionais poderão criar estratégias educativas para esclarecer possíveis mitos e estigmas sobre a doença e terapia, principalmente aquelas influenciadas pelas representações sociais de letalidade do câncer, que limitam a esperança e o investimento de recursos internos e externos desses pais. Dessa forma, destaca-se o papel dos profissionais na abordagem individualizada, de acordo com as particularidades da doença, da criança e da família, para sanar as dúvidas, esclarecer questões de tratamento e fortalecer os vínculos e a perspectiva do desfecho relacionado à doença. Este estudo ainda tem o potencial de abrir caminhos para futuras pesquisas que abranjam a influência das variações culturais sobre essas concepções, mitos e estigmas vinculados ao câncer infantil, além de chamarem a atenção para a necessidade de novas investigações sobre uma temática ainda pouco explorada na literatura.

\section{Referências}


1. Instituto Nacional de Câncer José Alencar Gomes da Silva (INCA). Estimativa 2018: incidência de câncer no Brasil [Internet]. Rio de Janeiro (RJ): Inca; 2018 [acesso em 2019 dez 13]. Disponível em: https://www.inca.gov.br/sites/ufu.sti.inca.local/files//media/document//estimativa-incidencia-de-cancerno-brasil-2018.pdf

2. Instituto Nacional de Câncer José Alencar Gomes da Silva (INCA). Incidência, mortalidade e morbidade hospitalar por câncer em crianças, adolescentes e adultos jovens no Brasil: informações dos registros de câncer e do sistema de mortalidade [Internet]. Rio de Janeiro (RJ): Inca; 2016 [acesso em 2018 dez 10]. Disponível em: http://www1.inca.gov.br/wcm/incidencia/2017/pdf/versao-completa.pdf

3. Rodgers CC, Stegenga K, Withymcombe JS, Sachse K, Kelly KP. Processing information after a child's cancer diagnosis - how parents learn. J Pediatr Oncol Nurs [Internet]. 2016 [acesso em 2020 jan 13];33(6):447-59. Disponível em: https://www.ncbi.nlm.nih.gov/pubmed/28084180 doi: $10.1177 / 1043454216668825$

4. Di Battista A, Dupuis LL, Cassidy M, Portwine C, Johnston DL, Pradier Silva M, et al. Parent attributions about child symptoms related to cancer therapy. J Pediatr Oncol Nurs [Internet]. 2017 [acesso em 2018 jun 08 ];34(1):44-50. Disponível em: https://www.ncbi.nlm.nih.gov/pubmed/26825448 doi: $10.1177 / 1043454215628002$

5. Licqurish S, Phillipson L, Chiang P, Walker J, Walter F, Emery J. Cancer beliefs in ethnic minority populations: a review and meta-synthesis of qualitative studies. Eur J Cancer Care (Engl) [Internet]. 2017 [acesso em 2018 jun 20];26(1):1-13. Disponível em: https:/www.ncbi.nlm.nih.gov/pubmed/27515153 doi: 10.1111/ecc.12556

6. Cox T. Caregivers reflecting on the early days of childhood cancer. Eur J Cancer Care (Engl) [Internet]. 2017 [acesso em 2018 jun 22 ];27(1):1-10. Disponível em: https://www.ncbi.nlm.nih.gov/pubmed/27094577 doi: 10.1111/ecc.12499

7. Kelly KP, Withycombe J, Stegenga K, Rodgers C. The why behind the questions: question-asking in parents of children newly diagnosed with cancer - a report from the Children's Oncology Group. J Pediatr Oncol Nurs [Internet]. 2018 [acesso em 2020 jan 13];43:23-8. Disponível em: https://www.ncbi.nlm.nih.gov/pubmed/30473153 doi: 10.1016/j.pedn.2018.07.002

8. Leventhal H, Brissette I, Leventhal EA. The commonsense model of self-regulation of health and illness. In: Cameron LD, Leventhal H. The self-regulation of health and illness behavior. New York: Routledge; 2003. p. 42-65.

9. Elo S, Kyngas H. The qualitative content analysis process. J Adv Nurs [Internet]. 2008 [acesso em 2018 abr 06];62(1):107-15. Disponível em: https://www.ncbi.nlm.nih.gov/pubmed/18352969 doi: 10.1111/j.13652648.2007.04569.x 
10. Machado GOC, Biancovilli P, Jurberg C. Voices about a stigma: cancer in the opinion of three different segments in Brazilian society. J Can Educ [Internet]. 2017 [acesso em 2018 abr 06]; 2017 [acesso em 2018 abr 06];32(2):413-6. Disponível em: https://www.ncbi.nlm.nih.gov/pubmed/26676405 doi: 10.1007/s13187-015-0962-5

11. Nair M, Paul LT, Latha PT, Parukkutty K. Parents' knowledge and attitude regarding their child's cancer and effectiveness of initial disease counseling in pediatric oncology patients. Indian J Palliat Care [Internet]. 2017 [acesso em 2018 abr 10];23(4):393-8. Disponível em: https://www.ncbi.nlm.nih.gov/pmc/articles/PMC5661340/ doi: 10.4103/IJPC.IJPC_83_17

12. Carreño-Moreno S, Díaz LC, Blanco-Sánchez P. Cuidador familiar del niño con cáncer: un rol en transición. Rev Latinoam Bioét [Internet]. 2017 [acesso em 2019 jan 29];17(2):18-30. Disponível em: http://www.scielo.org.co/scielo.php?script=sci_arttext\&pid=S1657-47022017000200018 \&lng=en doi 10.18359/rlbi.2781

13. Lawrenz P, Peuker AC, Castro EK. Illness perceptions and indicators of PTSD in mothers of childhood cancer survivors. Temas Psicol [Internet]. 2016 [acesso em 2018 nov 22];24(2):439-50. Disponível em: http://pepsic.bvsalud.org/pdf/tp/v24n2/en_v24n2a03.pdf doi: 10.9788/TP2016.2-03Pt

14. Lima BC, Silva LF, Goes FGB, Ribeiro MTS, Alves LL. O itinerário terapêutico de famílias de crianças com câncer: dificuldades encontradas neste percurso. Rev Gaúch Enferm [Internet]. 2018 [acesso em 2018 nov 22];39:1-9. Disponível em: http://www.scielo.br/scielo.php?script=sci_arttext\&pid=S198314472018000100455 doi: 10.1590/1983-1447.2018.20180004

15. Silva-Rodrigues FM, Moraes KSR, Duarte AM, Polita NB, Padula MPC, Nascimento LC. Información a los padres durante el tratamiento del cáncer infantil: un studio descriptivo. Cult Cuid [Internet]. 2019 [acesso em 2020 jan15];54:297-309. Disponível em: https://rua.ua.es/dspace/bitstream/10045/96336/1/CultCuid_54-297-309.pdf doi: 10.14198/cuid.2019.54.26

16. Marron JM, Cronin AM, Kang TI, Mack JM. Intended and unintended consequences: ethics, communication, and prognostic disclosure in pediatric oncology. Cancer [Internet]. 2018 [acesso em 2020 jan 15];124(6):1232-41. Disponível em: https://www.ncbi.nlm.nih.gov/pubmed/29278434 doi: $10.1002 /$ cncr.31194

17. Koch KD, Jones BL. Supporting parent caregivers of children with life-limiting illness. Children [Internet]. 2018 [acesso em 2020 jan 15];5(7):85-92. Disponível em: https://www.ncbi.nlm.nih.gov/pmc/articles/PMC6069074/ doi: 10.3390/children5070085

18. Nicholas DB, Barrera M, Granek L, D'Agostino NM, Shaheed J, Beaune L, et al. Parental spirituality in life-threatening pediatric cancer. J Psychosoc Oncol [Internet]. 2017 [acesso em 2018 maio 15];35(3):323-34. Disponível em: https:/www.ncbi.nlm.nih.gov/pubmed/28300487 doi: 10.1080/07347332.2017.1292573 
17 | Silva-Rodrigues, FM; Vulczak VLS; Alencar CF; Santos LSC; Nascimento LC

19. Crisp CL. Faith, hope, and spirituality: supporting parents when their child has a life-limiting illness.

J Christ Nurs [Internet]. 2016 [acesso em 2018 jan 10];33(1):14-21. Disponível em: https://www.ncbi.nlm.nih.gov/pubmed/26817365

\section{Autor correspondente}

Fernanda Machado Silva-Rodrigues

E-mail: fernanda.rodrigues@fcmsantacasasp.edu.br

Endereço: Rua Dr. Cesário Mota Junior, 61 - CEP 01221-020 - Vila Buarque - São Paulo - SP

\section{Contribuições de Autoria}

\section{1 - Fernanda Machado Silva-Rodrigues}

Concepção e desenvolvimento do projeto, análise e discussão dos dados e redação final do artigo

\section{2 - Verônica Leticia da Silva Vulczak}

Desenvolvimento do projeto, análise e discussão dos dados

\section{3 - Carolina Fonseca Alencar}

Contribuições: Coleta, análise e discussão dos dados

\section{4 - Luciana Soares Costa Santos}

Redação do artigo e revisão crítica

5 - Lucila Castanheira Nascimento

Redação do artigo e revisão crítica

\section{Como citar este artigo}

Silva-Rodrigues FM, Vulczak VLS, Alencar CF, Santos LSC, Nascimento LC. Concepções dos pais acerca da doença oncológica e do tratamento quimioterápico de seus filhos. Rev. Enferm. UFSM. 2020 [Acesso em: Anos Mês Dia]; vol.10 e33: 1-17. DOI: https://doi.org/10.5902/2179769235898 